\title{
General relativistic, nonstandard model for the dark sector of the Universe
}

\author{
P. C. Stichel ${ }^{1, \mathrm{a}}$, W. J. Zakrzewski ${ }^{2, \mathrm{~b}}$ \\ ${ }^{1}$ Fakultät für Physik, Universität Bielefeld, 33501 Bielefeld, Germany \\ ${ }^{2}$ Department of Mathematical Sciences, University of Durham, Durham DH1 3LE, UK
}

Received: 12 September 2014 / Accepted: 4 December 2014 / Published online: 14 January 2015

(C) The Author(s) 2014. This article is published with open access at Springerlink.com

\begin{abstract}
We present a general relativistic version of the self-gravitating fluid model for the dark sector of the Universe (darkon fluid) introduced in Stichel and Zakrzewski (Phys Rev D 80:083513, 2009) and extended and reviewed in Stichel and Zakrzewski (Entropy 15:559, 2013). This model contains no free parameters in its Lagrangian. The resulting energy-momentum tensor is dustlike with a nontrivial energy flow. In an approximation valid at sub-Hubble scales we find that the present-day cosmic acceleration is not attributed to any kind of negative pressure but it is due to a dynamically determined negative energy density. This property turns out to be equivalent to a time-dependent spatial curvature. The obtained cosmological equations, at sub-Hubble scales, agree with those of the nonrelativistic model but they are given a new physical interpretation. Furthermore, we have derived the self-consistent equation to be satisfied by the nonrelativistic gravitational potential produced by a galactic halo in our model from a weak-field limit of a generalized TolmanOppenheimer-Volkoff equation.
\end{abstract}

\section{Introduction}

It is now pretty clear that the present Universe undergoes a phase of accelerated expansion (see the recent reviews [1,2]). On the other hand there exists overwhelming evidence for the existence of gravitational effects on all cosmological scales (termed "dark matter") which cannot be explained by the gravitation of standard matter in the framework of general relativity (see the review [3]). All of these data are in good agreement with a $\Lambda$-cold dark matter (CDM) cosmology (see $[1,2]$ and the literature cited therein). But this $\Lambda$ CDM model suffers, at least, from the following insufficiencies:

\footnotetext{
a e-mail: peter@physik.uni-bielefeld.de

be-mail:w.j.zakrzewski@durham.ac.uk
}

- Interpreted as the energy density of the vacuum the experimental value of $\Lambda$ turns out to be too small by a factor of $10^{54}$ (see [4]).

- None of the proposed DM-constituents has been observed (cp. [5]).

- There is a CDM-controversy on small scales [6].

Other observations which are in disagreement with the $\Lambda \mathrm{CDM}$ model have been recently listed by Kroupa [7].

One can find in the literature a large number of papers explaining either the accelerated expansion and/or dark matter by changing either the geometrical part of Einstein's field equations (EFEs) (termed modified gravity) or the matter part (addition of some scalar and/or tensor fields). We will not comment on either of these attempts (for details see e.g. the reviews $[1,2]$ and the literature cited therein). But we want to point out that all these proposals are of a phenomenological nature, they contain either some new parameters or even free functions. To overcome this freedom we need some new (i.e. unconventional) physics which, however, should be based on known physical principles (e.g. symmetry). Such a model containing no new constants in its Lagrangian and based on Galilean symmetry (minimal gravitational coupling of massless Galilean particles in agreement with the equivalence principle) has been presented in [8], further developed in $[9,10]$ and reviewed and extended in [11]. This nonrelativistic, unified model for the dark sector of the Universe is an exotic fluid model, termed darkon fluid model, which contains beside the standard hydrodynamic fields also a new vector field $\vec{q}(\vec{x}, t)$. This model describes successfully observational data for the transition from a decelerating to an acceleration phase of the Universe as well as the flat behavior of galactic rotation curves $[8,11]$.

The aim of the present paper is to present a general relativistic version of this model and to relate some approximate solutions of it to the corresponding solutions of its nonrelativistic counterpart. 
The paper is organized as follows: to get a self-consistent paper and to have an appropriate starting point for its relativistic generalization we present in Sect. 2 a short review of the nonrelativistic model [8-11]. In Sect. 3 we treat first the special-relativistic generalization of the free model, discuss the different options to consider classical spin contributions and, after a Belinfante transformation, we introduce gravitation by the principle of minimal coupling. Also we discuss the energy conditions. In Sect. 4 we consider the dynamics of the coupled system of the Einstein field equations and the relativistic darkon fluid equations of motion in spherical geometry. Solutions of these equations at sub-Hubble scales which agree with the cosmological solutions obtained by the nonrelativistic model are treated in Sect. 5. We show that these cosmological solutions turn out to be completely different from those of the Friedmann-Lemaitre-RobertsonWalker (FLRW) model. In Sect. 6 we treat the same coupled system of equations in non-comoving coordinates and derive the self-consistent equation for the halo-gravitational potential derived in [11], as a weak-field limit of the TolmanOppenheimer-Volkoff equation. Some final remarks are presented in Sect. 7.

\section{Nonrelativistic, self-gravitating darkon fluid}

In [8] we have introduced nonrelativistic massless 'particles' as a dynamical realization of the unextended Galilei group. These 'particles' move in an enlarged twelve-dimensional phase space [11] consisting of

- the 'particle' trajectory $\vec{x}(t)$

- the momentum $\vec{p}(t)$, canonically conjugate to $\vec{x}(t)$

- the velocity vector $\vec{y}(t)$ and

- the reduced boost vector $\vec{q}(t)$ (called 'pseudo-coordinate'), canonically conjugate to $\vec{y}(t)$.

In accordance with the Galilean algebra the corresponding 'one-particle' Hamiltonian $H$ is given by

$H_{0}=p_{i} y_{i}$,

corresponding to, by a Legendre transformation, the Lagrangian

$L_{0}=p_{i}\left(\dot{x}_{i}-y_{i}\right)+q_{i} \dot{y}_{i}$

and so giving the equations of motion (EOMs):

$\dot{x}_{i}=y_{i}, \quad \dot{p}_{i}=0, \quad \dot{q}_{i}=-p_{i}, \quad \dot{y}_{i}=0$.

But such a 'particle' is not a classical particle in the usual sense as it is not detectable by any finite-sized macroscopic measurement device because
- momentum and velocity vector are independent of each other and we have no ability to measure the momentum,

- the boost vector $\vec{q}$ has, for fixed position $\vec{x}$ and velocity $\vec{y}$ an arbitrary i.e. undetermined length.

For these reasons we have called these 'particles' darkons $[9,10]$, as they exist only as elements of an exotic fluid whose self-gravitating version is a substitute for what is usually called 'dark energy' and 'dark matter'.

To introduce the coupling to gravitation represented by the field strength $g_{i}(\vec{x}, t)$ we have to require, in agreement with Einstein's equivalence principle, the validity of Newton's law

$\ddot{x}_{i}(t)=g_{i}(\vec{x}(t), t)$,

which will be realized if we add to $L_{0}$ an interaction part (minimal coupling)

$L_{\text {int }}=-q_{i} g_{i}$.

An important property of our darkons is the appearance of a macroscopic spin: the conserved total angular momentum is given by the sum of the usual orbital angular momentum and a second term which we call, for convenience, spin [11] (see also Mathisson [12])

$J_{i}=\epsilon_{i k l}\left(x_{k} p_{l}+y_{k} q_{l}\right)$.

Note that the two terms in (5) act separately as generators of rotations in the $\{\vec{x}, \vec{p}\}$ and $\{\vec{y}, \vec{q}\}$ parts of the phase space.

Now the question arises whether we can find some a simple physical system which mimics one darkon coupled to an external gravitational field. To get this we start with the second order Lagrangian

$L=-\dot{q}_{i} \dot{x}_{i}$,

which has been obtained from the Lagrangian (2) by the elimination of the momentum $p_{i}$. By introducing the point transformation

$\left(x_{i}, q_{i}\right) \rightarrow\left(x_{i}^{+}, x_{i}^{-}\right), \quad$ with $x_{i}^{ \pm} \equiv x_{i} \pm \frac{q_{i}}{2 m_{0}}$,

where $m_{0}$ is a free mass-parameter introduced for dimensional reasons, we obtain

$L=\frac{m_{0}}{2}\left(\left(x_{i}^{-}\right)^{2}-\left(x_{i}^{+}\right)^{2}\right)$.

This is a system of two non-interacting point particles with their masses having opposite sign but equal magnitude. The coordinates $x_{i}$ describe the motion of the geometric center and $q_{i} / m_{0}$ describe the relative motion of the two particles (therefore we have called $q_{i}$ 'pseudo-coordinate') So, the vanishing mass of free darkons comes about by the cancelation of two mass terms.

To arrive at the interaction Lagrangian (5) we start with the standard expression for the interaction of two massive particles with an external gravitational potential, 
$L_{\text {int }}=-m_{0} \phi\left(\vec{x}^{-}\right)+m_{0} \phi\left(\vec{x}^{+}\right)$.

If we insert the expressions for $x_{i}^{ \pm}$from (8) and perform a Taylor expansion about $x_{i}$ we obtain, in lowest order,

$L_{\text {int }}=q_{i} \partial_{i} \phi+\cdots$,

which agrees exactly with our ansatz (5) if the field strength $g_{i}$ is given, as usual, by the gradient of a potential $g_{i}=-\partial_{i} \phi$.

We need to add a word of caution: the description just given cannot be understood as a derivation of the Lagrangian (5) because the length of the vector $q_{i}$ is unbounded and it is not small when compared to the length of the coordinate vector $x_{i}$. So the physical picture given above serves only for illustrative purposes.

To promote the 'one-particle' picture to a self-gravitating fluid we replace the 'one-particle' phase space coordinates $A_{i}=\left\{x_{i}, p_{i}, q_{i}, y_{i}\right\}$ by the continuum labeled by $\vec{\xi} \in R^{3}$ (comoving coordinates) $A_{i}(t) \rightarrow A_{i}(\vec{\xi}, t)$.

The Lagrangian for our darkon fluid then becomes

$L=\int \mathrm{d}^{3} \xi\left[p_{i}\left(\dot{x}_{i}-y_{i}\right)+q_{i}\left(\dot{y}_{i}-g_{i}\right)\right]+L_{\text {field }}$

where, as usual,

$L_{\text {field }}=-\frac{1}{8 \pi G} \int \mathrm{d}^{3} x g_{i}^{2}(\vec{x}, t)$.

The Lagrangian (12) is invariant w.r.t. infinitesimal relabeling transformations $\vec{\xi} \rightarrow \vec{\xi}+\vec{\alpha}(\vec{\xi})$ with $\vec{\nabla}_{\xi} \cdot \vec{\alpha}=0$ leading to the conservation law [9-11]

$\dot{\theta}_{i}=0 \quad$ where $\theta_{i} \equiv-\frac{\partial \dot{x}_{k}}{\partial \xi_{i}} q_{k}+\frac{\partial x_{k}}{\partial \xi_{i}} \dot{q}_{k}$,

which, after elimination of the momentum field $p_{i}$, allows us to reduce the EOM for $q_{i}$ to a first order equation [9-11]. Then by means of the usual transformations from comoving coordinates $\vec{\xi}$ to the fixed ones $\vec{x}=\vec{x}(\vec{\xi}, t)$ we obtain from the Lagrangian formulation (12) the Eulerian formulation given by the Lagrangian [11]

$L=\int \mathrm{d}^{3} x\left[n q_{i}\left(D_{t} u_{i}-g_{i}\right)-\theta\left(\dot{n}+\partial_{k}\left(n u_{k}\right)\right)+n \alpha D_{t} \beta\right]$ $+L_{\text {field }}$

where $n(x, t)$ denotes the 'particle' density. We have introduced the auxiliary field

$\left.\theta_{i}(\vec{x}, t) \equiv \frac{\partial \xi_{k}}{\partial x_{i}} \theta_{k}(\vec{\xi})\right|_{\vec{\xi}=\vec{\xi}(\vec{x}, t)}$

and its Clebsch parameterization $\theta_{i}=\partial_{i} \theta+\alpha \partial_{i} \beta$.

Furthermore, $\vec{u}$ denotes the velocity field $u_{k}(\vec{x}, t) \equiv$ $\left.\dot{x}_{k}(\vec{\xi}, t)\right|_{\vec{\xi}=\vec{\xi}(\vec{x}, t)}$ and $D_{t}$ the convective derivative $D_{t} \equiv$ $\frac{\partial}{\partial t}+u_{k} \frac{\partial}{\partial x_{k}}$.

Note that the Hamiltonian corresponding to the Lagrangian (15) is not bounded from below. In [8] we have argued that this does not lead to any stability problems.
The EOMs following from the Lagrangian (15) have been solved for:

- the isotropic, homogeneous case (cosmology) in [8], respectively, [11] (see also Sect. 5 of this paper),

- the spherically symmetric, steady state case modeling halos [11] (see also Sect. 6 of this paper).

\section{General relativistic approach}

3.1 Nongravitating, special-relativistic case

We start our discussion with the nongravitating i.e. special relativistic case for two reasons:

- to discuss the notion of (zero) rest-mass in our enlarged phase space,

- to discuss the role of the spin-term within the energymomentum tensor (EMT).

The relativistic generalization of the action corresponding to the free matter part of the Lagrangian (15) is then given by [we use the Minkowski metric $\eta_{\mu \nu}=$ $\operatorname{diag}(-1,+1,+1,+1)]$

$S=\int \mathrm{d}^{4} x\left(n q_{\nu} D u^{\nu}-\theta \partial_{\nu}\left(n u^{\nu}\right)+n \alpha D \beta\right)$,

where we have defined the relativistic version of the convective derivative by $D \equiv u^{\lambda} \partial_{\lambda}$. We also require that the velocity field $u^{v}$ obeys the usual constraint $u_{v} u^{v}=-1$.

From the Lagrangian (16) we derive the Euler-Lagrange EOMs

$\partial_{\nu}\left(n u^{\nu}\right)=0, \quad D u^{\nu}=0$,

$D \alpha=D \beta=D \theta=0, \quad D q_{\lambda}=q_{\nu} \partial_{\lambda} u^{\nu}+\theta_{\lambda}$

with

$\theta_{\lambda} \equiv \partial_{\lambda} \theta+\alpha \partial_{\lambda} \beta$

Then the first part of the EOMs (18) is equivalent to the EOM

$D \theta_{\lambda}+\theta_{\nu} \partial_{\lambda} u^{\nu}=0$

with the constraint

$u^{\lambda} \theta_{\lambda}=0$.

It is easy to see that

- the four-momentum vector field, defined analogously to the EOM $p_{i}=-\dot{q}_{i}$ in (3) by $p_{\mu} \equiv-D q_{\mu}$, is space-like (from the EOMs one deduces that $p^{\lambda} u_{\lambda}=0$ ), 
- the second EOM in (18) is invariant w.r.t. the gauge transformation $q_{\lambda} \rightarrow q_{\lambda}+\epsilon u_{\lambda}$, i.e. we can fix the gauge by choosing $q_{\lambda} u^{\lambda}=0$ so that $q_{\lambda}$ becomes space-like,

- the EOMs are invariant w.r.t. the shift symmetry $q_{\lambda} \rightarrow$ $q_{\lambda}+c_{\lambda}, \theta \rightarrow \theta-c_{\lambda} u^{\lambda}$, where $c_{\lambda}$ is a constant vector field. Note that this kind of shift symmetry is characteristic for so called Galileon theories (cp. section 2.1 in [13]).

The fact that $p_{\mu}$ is a space-like vector field could easily lead to the wrong conclusion: that our darkons are tachyons (cp. appendix B in [8]). But, as argued by Weyssenhoff and Raabe [14] in a similar context, we should define the restmass as the energy in the rest system of the 'particle' given by $m_{0}=-u^{\lambda} p_{\lambda}$, which, however, vanishes in our case.

The Poincare invariance leads to the existence of two conserved currents (cp. Appendix A in [15])

- From translational invariance we get the canonical, nonsymmetric EMT

$$
\hat{T}^{\mu \nu}=n p^{\mu} u^{v}
$$

- From Lorentz invariance we get

$$
J^{\nu, \alpha \beta}=x^{\alpha} \hat{T}^{\beta v}-x^{\beta} \hat{T}^{\alpha v}+S^{\nu, \alpha \beta},
$$

where the spin tensor is given in our case by

$$
S^{\nu, \alpha \beta}=n u^{\nu}\left(u^{\alpha} q^{\beta}-u^{\beta} q^{\alpha}\right)
$$

The conservation law $\partial_{\nu} \hat{T}^{\mu \nu}=0$ follows immediately from the EOMs. Furthermore, the EOMs also give us

$\partial_{\nu} S^{\nu, \alpha \beta}=\hat{T}^{\alpha \beta}-\hat{T}^{\beta \alpha}$

and so yield

$\partial_{\nu} J^{\nu, \alpha \beta}=0$.

Note that the relativistic fluid described by the action (16) is a spin fluid which, as usually, is described by a scalar density $n$, a four-velocity $u^{\mu}$ and an anti-symmetric spin tensor $S^{\alpha \beta}$ defined in our case by [see Eq. (24)]

$S^{\alpha \beta}=n\left(u^{\alpha} q^{\beta}-u^{\beta} q^{\alpha}\right)$.

Contrary to the standard relativistic spin fluid, our spin tensor (26) does not obey the Frenkel condition [16]

$S^{\alpha \beta} u_{\beta}=0$,

nor any other spin supplementary condition (for an exhaustive discussion of all these conditions see e.g. [17]). It is an important property of our fluid that we do not need such a supplementary condition as the dynamics of the spin tensor (26) is completely fixed by the EOMs for $n, u^{\mu}$ and the field $q^{\mu}$. But for a standard relativistic fluid we need, besides the conservation law $\partial_{\nu} T^{\mu \nu}=0$, also three additional equations to obtain a well-determined system. These additional equations are just the spin supplementary conditions [e.g. the Frenkel condition (27)].

\subsection{General relativistic dynamics}

According to Hehl [18] we have now two possibilities for coupling our relativistic fluid to gravity.

- To gauge away the spin tensor by a Belinfante transformation [19]

$$
\hat{T}^{\mu \nu} \rightarrow T^{\mu \nu}=\hat{T}^{\mu \nu}+\frac{1}{2} \partial_{\lambda}\left(S^{\mu, \nu \lambda}+S^{\lambda, \nu \mu}+S^{\nu, \mu \lambda}\right)
$$

leading to a conserved, symmetric EMT

$$
T^{\mu \nu}=n\left(u^{\mu} p^{\nu}+u^{\nu} p^{\mu}\right)+\partial_{\lambda}\left(n u^{\mu} u^{\nu} q^{\lambda}\right) .
$$

This EMT may then be used as a source term in EFEs after we have performed the substitutions (30) [see Eq. (38)].

- Consider spin as a dynamical variable by relating the spin tensor to the torsion tensor in the framework of a RiemannCartan space-time and use the canonical EMT (22) as the source term in EFEs.

In this paper we prefer to use the first possibility as in this case we can reproduce, at sub-Hubble scales, the cosmological equations which are valid for the nonrelativistic darkon fluid (Sect. 5). To realize this we have to apply the principle of minimal gravitational coupling (cp. [20]): so we perform the substitutions

$\eta_{\mu \nu} \rightarrow g_{\mu \nu}$ and $\quad \partial_{\lambda} \rightarrow \nabla_{\lambda}$

in the special-relativistic action (16). Here $g_{\mu \nu}$ is the metric tensor and $\nabla_{\lambda}$ is the covariant derivative $\nabla_{\lambda} A^{\nu}=\partial_{\lambda} A^{\nu}+$ $\Gamma_{\lambda \sigma}^{\nu} A^{\sigma}$ where the elements of the connection $\Gamma_{\lambda \sigma}^{\nu}$ are given by the Christoffel symbols.

To obtain also EFEs from the principle of least action we have to consider the total action

$S=\int \mathrm{d}^{4} x \sqrt{-g}\left(n q_{\nu} u^{\lambda} \nabla_{\lambda} u^{\nu}-\theta \nabla_{\nu}\left(n u^{\nu}\right)+n \alpha u^{\lambda} \partial_{\lambda} \beta\right)$

$+S_{\mathrm{EH}}$

with the Einstein-Hilbert action $S_{\mathrm{EH}}$ given by the wellknown expression

$S_{\mathrm{EH}}=\frac{1}{16 \pi G} \int \mathrm{d}^{4} x \sqrt{-g} R$,

where $g$ is the determinant of $g_{\mu \nu}, R$ is the Ricci scalar, $n$ is the particle density, and $u^{\lambda}$ and $q^{\lambda}$ are the velocity field 
and the relativistic generalization of the pseudo-coordinate field. The scalar fields $\theta, \alpha$ and $\beta$ are Lagrange-multiplier fields which originate from the relabeling symmetry (see the nonrelativistic Lagrangian formulation in Sect. 2).

From the action (31) we derive the darkon fluid EOMs [which, alternatively, may be obtained by applying the substitution rule (30) to the special-relativistic EOMs (17-21)]

$\nabla_{\lambda}\left(n u^{\lambda}\right)=0, \quad u^{\lambda} \nabla_{\lambda} u^{\nu}=0$

$u^{\lambda} \nabla_{\lambda} q_{v}=q_{\lambda} \nabla_{\nu} u^{\lambda}+\theta_{\nu}$

and

$u^{\lambda} \nabla_{\lambda} \theta_{v}+\theta_{\lambda} \nabla_{v} u^{\lambda}=0 \quad$ with $u^{v} \theta_{v}=0$

where by (19) $\theta_{\nu} \equiv \partial_{\nu} \theta+\alpha \partial_{\nu} \beta$ and EFEs are the standard ones

$G^{\mu \nu} \equiv R^{\mu \nu}-\frac{1}{2} g^{\mu \nu} R=8 \pi G T^{\mu \nu}$.

Here $R^{\mu \nu}$ is the Ricci tensor and the EMT $T^{\mu \nu}$ is given by (38) given below.

We find again that the fields $q_{\lambda}$ and $p_{\lambda} \equiv-u^{\nu} \nabla_{\nu} q_{\lambda}$ obey the constraints

$u^{\lambda} q_{\lambda}=u^{\lambda} p_{\lambda}=0$

and so they are space-like (recall that $u^{\lambda}$ is time-like, normalized by $u^{\lambda} u_{\lambda}=-1$ ).

\subsection{Energy-momentum tensor (EMT)}

The EMT (29), after having performed the substitutions (30), becomes

$T^{\mu \nu}=-n\left[u^{\mu} u^{\lambda} \nabla_{\lambda} q^{v}+(\mu \leftrightarrow v)\right]+\nabla_{\lambda}\left(n u^{\mu} u^{v} q^{\lambda}\right)$.

By using the darkon fluid EOMs the expression (38) can be brought into its canonical form (see [21])

$T^{\mu \nu}=\rho u^{\mu} u^{\nu}+k^{\mu} u^{\nu}+k^{v} u^{\mu}$,

where for our model

$\rho=\nabla_{\lambda}\left(n q^{\lambda}\right)$ and $k^{\mu}=n\left(q^{\lambda}\left(\partial_{\lambda} u^{\mu}-\partial^{\mu} u_{\lambda}\right)-\theta^{\mu}\right)$

are the energy density and the energy-flow vector seen by an observer comoving with the darkon fluid.

Usually the vector $k^{\mu}$ is called the 'heat-flow vector'. But such a terminology assumes, at least implicitly, that we have a description of $k^{\mu}$ and $\rho$ in terms of a relativistic, irreversible thermodynamics (for the general framework see [22], for an application to cosmology see [23]). But $\rho$ and $k^{\mu}$ are completely fixed in our case by the darkon fluid EOMs. So it is an open question whether they are accessible to a thermodynamic description or whether the arising energy flow is due to the generation of gravitational radiation. It is outside the scope of the present paper to consider this question.

Note that the expression (40) for the energy density $\rho$ is not positive definite! So at least the weak energy condition is violated. But, as will be shown in Sect. 5, exactly this property of our model is crucial for the model's explanation of the present-day accelerated expansion of the Universe. Energy conditions are constraints on the EMT of a general relativistic fluid which, originally, has been thought of as being necessary for the fluid 'to be physically reasonable' (see [24] and the literature cited within). But it is well known that e.g. the introduction of 'dark energy' within the FLRW model (negative pressure with $\rho+3 p<0$ ) violates the strong energy condition. This is in agreement with a very recent and general discussion in the framework of extended theories of gravitation [25], which comes to the conclusion that the violation of energy conditions is a general property in the presence of dark energy.

\section{Dynamics in spherically symmetric geometry}

The non-accelerated fluid (geodesic) motion (33) allows the consideration of synchronous comoving $\left(u^{\mu}=\delta_{0}^{\mu}\right)$, spherically symmetric coordinates defined by the metric

$\mathrm{d} s^{2}=-\mathrm{d} t^{2}+B^{2}(t, r) \mathrm{d} r^{2}+Y^{2}(t, r) \mathrm{d} \Omega^{2}$.

For this metric the space-like vectors $q_{\mu}$ and $\theta_{\mu}$ have only a non-vanishing radial component

$q_{\mu}=q s_{\mu}, \quad \theta_{\mu}=\tilde{\theta} s_{\mu} \quad$ with $s_{\mu} \equiv(0, B) ;$

here and in the following, the first component of a 2-dim vector describes the time-component and the second one the radial component.

The darkon fluid equations (33), (34), and (35) have the following form, respectively, solutions:

$n(t, r)=\frac{n_{0}(r)}{B Y^{2}}, \quad\left(\frac{q}{B}\right)=\frac{\alpha(r)}{B^{2}}, \quad \tilde{\theta}(t, r)=\frac{\alpha(r)}{B}$,

where $n_{0}(r)$ and $\alpha(r)$ are arbitrary integration functions. The energy density $\rho$ defined by (40), then becomes, in terms of $q$ and the metric

$\rho=\frac{1}{B Y^{2}}\left(\frac{n_{0} q}{B}\right)$

and obeys, due to the second equation in (43), the local energy conservation equation

$\dot{\rho}+\rho\left(\frac{\dot{B}}{B}+2 \frac{\dot{Y}}{Y}\right)-\frac{1}{B Y^{2}}\left(\frac{\alpha(r) n_{0}(r)}{B^{2}}\right)^{\prime}=0 ;$

here and in what follows ' denotes the derivative w.r.t. $r$.

Note that the velocity field $u^{\mu}$ has vanishing vorticity in the spherically symmetric case. Therefore the energy-flow vector $k^{\mu}$ reduces to 
$k^{\mu}=-n \theta^{\mu}$.

The Einstein field equations (36) now become (cp. eq. (7) with $A=1$ in [26])

$$
\begin{aligned}
& 2 \frac{\dot{B}}{B} \frac{\dot{Y}}{Y}+\frac{1+\dot{Y}^{2}}{Y^{2}}-\frac{Y^{\prime 2}}{Y^{2} B^{2}}-\frac{2}{Y B}\left(\frac{Y^{\prime}}{B}\right)^{\prime} \\
& -\frac{\kappa}{B Y^{2}}\left(\frac{n_{0} q}{B}\right)^{\prime}=0, \\
& 2 \frac{\ddot{Y}}{Y}+\frac{1+\dot{Y}^{2}}{Y^{2}}-\frac{Y^{\prime 2}}{Y^{2} B^{2}}=0, \\
& \frac{\ddot{B}}{B}+\frac{\ddot{Y}}{Y}+\frac{\dot{B} \dot{Y}}{B Y}-\frac{1}{B Y}\left(\frac{Y^{\prime}}{B}\right)^{\prime}=0, \\
& -\frac{\kappa}{2} n_{0}(r) \alpha(r)=Y B^{2}\left(\frac{Y^{\prime}}{B}\right),
\end{aligned}
$$

with $\kappa \equiv 8 \pi G$ where (47), (48), (49), and (50) represent, respectively, the $00, r r$, tangential and $0 r$-components of (36).

As a consequence of the covariant conservation of the Einstein tensor

$$
\nabla_{\nu} G^{\mu \nu}=0
$$

the four EFEs (47-50) are not independent of each other. Explicit calculations lead to the following results:

- The third EFE (49) is a consequence of the second and the fourth EFEs (48) and (50).

- The time derivative of $B Y^{2} \times 1$.h.s. (47) vanishes as a consequence of the other EFEs and the second EOM in (43).

These dependencies give rise to consistency relations which have to be respected if we consider approximate solutions of the EFEs (see Sect. 5).

For the discussion of approximate cosmological equations (see Sect. 5) it is also advantageous to express at least partially the EFEs in terms of some kinematic, respectively, geometric quantities. Kinematic quantities are defined by the EMT (see (39) with (46)) and the decomposition of the four-velocity gradient [21]

$\nabla_{\nu} u_{\mu}=\sigma_{\nu \mu}+\frac{1}{3} \hat{\theta} h_{\mu \nu}$,

where $\hat{\theta} \equiv \nabla_{\nu} u^{\nu}$ is the volume expansion scalar and $\sigma_{\nu \mu}$ is the traceless shear tensor which for our metric (41) takes the form

$\sigma_{\nu \mu}=\sqrt{3} \sigma\left(s_{\nu} s_{\mu}-\frac{1}{3} h_{\nu \mu}\right)$

with $\sigma \equiv \frac{1}{\sqrt{3}}\left(\frac{\dot{B}}{B}-\frac{\dot{Y}}{Y}\right)$.

The tensor $h_{\nu \mu}=g_{\nu \mu}+u_{\nu} u_{\mu}$ projects onto the space orthogonal to the 4-velocity $u^{\mu}$. Note that in our case the decomposition (52) contains neither a vorticity nor an acceleration term.

As a geometric quantity we also introduce the spatial Ricci scalar ${ }^{3} R$ (for its definition see [27], section 1.3.5).

Next we consider the following equations, which are derived from the EFEs (47-50) (see [27,28]):

- The Raychaudhuri-Ehlers (RE) equation

$$
\frac{\ddot{a}}{a}=-\frac{2}{3} \sigma^{2}-\frac{4 \pi G}{3} \rho,
$$

where the generalized scale factor $a(t, r)$ is defined by

$$
\frac{\dot{a}}{a} \equiv \frac{1}{3} \hat{\theta}
$$

- The generalized Friedmann equation

$$
\dot{a}^{2}+\frac{a^{2}}{6}\left({ }^{3} R-2 \sigma^{2}\right)=\frac{8 \pi G \rho a^{2}}{3},
$$

or, if we define an effective spatial curvature $K_{\text {eff }}$ by

$$
K_{\mathrm{eff}}(t, r) \equiv \frac{a^{2}}{6}\left({ }^{3} R-2 \sigma^{2}\right),
$$

equation (56) becomes

$$
\dot{a}^{2}+K_{\mathrm{eff}}=\frac{8 \pi G \rho a^{2}}{3} .
$$

Note that we have $K_{\text {eff }}=(0, \pm 1)$ in the FLRW case.

- From these equations and the relation

$$
\nabla_{\nu} k^{\nu}=-\frac{1}{B Y^{2}}\left(\frac{n_{0} \alpha}{B^{2}}\right)^{\prime}
$$

which follows from (46) and the first and third EOM in (43), we easily derive the time derivative of $K_{\text {eff }}$

$\dot{K}_{\mathrm{eff}}=\frac{4}{9} a^{2} \hat{\theta} \sigma^{2}+\frac{8 \pi G a^{2}}{3 B Y^{2}}\left(\frac{n_{0} \alpha}{B^{2}}\right)^{\prime}$.

So we obtain the local energy conservation equation (45) in the form

$\dot{\rho}+3 \frac{\dot{a}}{a} \rho-\frac{3}{8 \pi G a^{2}} \dot{K}_{\text {eff }}+\frac{\hat{\theta} \sigma^{2}}{6 \pi G}=0$.

Note that these equations are not independent: the RE equation (54) follows by time differentiation of (58) and the use of (60) and (61).

What about the contribution of baryonic matter within our model? Suppose we describe baryonic matter, averaged over small scale inhomogeneities, by dust moving with the same 
four-velocity then the darkon fluid. Then our cosmological equations contain only the total energy density $\rho$ given by the sum of the darkon fluid and the baryonic dust contribution. We are unable to discriminate between both contributions to $\rho$. This can be seen as follows: to take into account baryonic matter we have to add to the EMT (39) a dust contribution $T_{\mu \nu}^{B}$ ( $\rho^{B}$ is the baryonic energy density),

$T_{\mu \nu}^{B}=\rho^{B} u_{\mu} u_{\nu}$,

which is separately covariantly conserved

$\nabla_{\rho} T_{\mu \nu}^{B} g^{\rho \mu}=0$.

Then $\rho^{B}$ obeys the local energy conservation equation

$\dot{\rho}^{B}+\rho^{B}\left(\frac{\dot{B}}{B}+2 \frac{\dot{Y}}{Y}\right)=0$,

and we have to add $\kappa \rho^{B}$ to the r.h.s. of the first Einstein field equation (47). So only the total energy density appears in (45). But the solution of the energy conservation equation (45) for the darkon fluid is only fixed modulo a solution of the corresponding homogeneous equation which is just given by (64).

Let us next show that, for isotropic coordinates,

$Y(t, r)=r B(t, r)$,

equations (48)-(50) enforce $\alpha(r)=0$ : By equating (48) and (49) in the isotropic case (65) we eliminate the time derivatives and obtain the well-known result (see [29])

$$
\left(\frac{1}{r}\left(\frac{1}{B}\right)^{\prime}\right)^{\prime}=0
$$

with the solution

$$
B(t, r)=\frac{a(t)}{1+\frac{r^{2}}{4} K(t)}
$$

where $a(t)$ and $K(t)$ are arbitrary functions of $t$. Now inserting (65), (67) into (48) leads by a straightforward calculation to $K(t)=K=$ const and, therefore, to the vanishing r.h.s. of (50). But then the EMT contains only a pure dust term which leads to a trivial cosmology (presence of only a decelerating phase).

\section{Solutions at sub-Hubble scales}

Unfortunately we are unable to solve Einstein's equations for $\alpha \neq 0$ exactly. So let us look for approximate solutions at sub-Hubble scales $\frac{r}{r_{0}}=\epsilon \ll 1$ ( $r_{0}=$ Hubble radius) and take correspondingly for the derivatives (cp. [30])

$\partial_{r}=O\left(\epsilon^{-1}\right)$ and $\partial_{t}=O\left(\epsilon^{-\frac{1}{2}}\right)$.
From (48) we obtain the exact relation

$$
B=\frac{Y^{\prime}}{(1-b)^{\frac{1}{2}}}
$$

with

$b=-\left(2 \ddot{Y} Y+\dot{Y}^{2}\right)$

Now we consider those metrics which have $Y(t, r) \propto r$ for small $r$. Then we have $b=O(\epsilon)$ and therefore $b$ may be treated as a perturbation.

Next we obtain, in leading order, from (50)

$\dot{b}(t, r)=8 \pi G \frac{n_{0} \alpha(r)}{Y Y^{\prime 2}}$,

which, when compared with (70), leads to the consistency relation

$-\left(\ddot{Y} Y^{2}\right)=4 \pi G \frac{n_{0} \alpha}{Y^{\prime 2}}$.

Then, in accordance with the interdependences of the EFEs described in Sect. 4, the third Einstein equation (49) is also fulfilled in leading order.

Instead of the first EFE (47), we use the RE equation (54) yielding, in leading order,

$2 \ddot{Y} Y^{\prime}+\ddot{Y}^{\prime} Y=-\frac{4 \pi G}{Y}\left(\frac{n_{0} q}{Y^{\prime}}\right)^{\prime}$,

which, after multiplication by $Y$, can be integrated to give

$\ddot{Y} Y^{2}=-4 \pi G \frac{n_{0} q}{Y^{\prime}}+f(t)$,

where $f(t)$ is an integration function. But if we differentiate (74) w.r.t. the time $t$ and use the EOM (43) for $\frac{q}{B}$, given in leading order by

$\left(\frac{q}{Y^{\prime}}\right)=\frac{\alpha}{Y^{\prime 2}}$,

we find, by comparison with the consistency relation (72), that $f$ must be a constant. To get an analytic solution we put $f$ equal to zero. So finally we have to solve the coupled system of equations (74) for $f=0$ and (75). To do this we consider a separation ansatz for $Y$,

$Y(t, r)=a(t) y(r)$,

leading by (74) to a separable form for $q$

$q(t, r)=q_{0}(t) q_{1}(r)$

where, due to (75), we may normalize $q_{1}$ so that

$q_{1}(r)=4 \pi G \frac{\alpha(r)}{y^{\prime}(r)}$.

Then we get for $q_{0}$ the equation

$\left(\frac{q_{0}}{a}\right)=\frac{1}{4 \pi G a^{2}}$. 
Finally from (74) we obtain

$\ddot{a} a^{3}=4 \pi G K_{1} q_{0}$

where $y^{3} y^{\prime 2}=-\frac{4 \pi G}{K_{1}} \alpha n_{0}$,

and $K_{1}$ is an arbitrary constant.

So the $r$-dependence of our solutions is completely specified by the choice of the integration functions $n_{0}(r)$ and $\alpha(r)$ (for the case of cosmology we refer to the next subsection). We note that the separable forms of $Y$ and $q$ lead also to a separable form for the energy density $\rho$ (44). Therefore the appearance of a perpetuum mobile of the third kind (continuous transfer of energy from one space region to another one) as advocated by Ivanov [31] is excluded.

\subsection{Connection with the nonrelativistic darkon fluid cosmology}

Note that (79) and (80) have exactly the form of the cosmological equations derived for the nonrelativistic darkon fluid in [8] and [11]. But which choice has to be made for the two free functions $n_{0}(r)$ and $\alpha(r)$ ?

For the cosmological solutions to be viable we have to require that the energy density $\rho$ as well as the darkon density $n$ are functions of time only. Now taking $\rho$ from (44) in leading order and using the ansätze (76) and (77) together with (81) we obtain

$\rho(t, r)=-\frac{K_{1} q_{0}(t)}{a^{4}(t) y^{\prime}(r) y^{2}(r)}\left(y^{3}(r) y^{\prime}(r)\right)^{\prime}$.

So to get $\rho=\rho(t)$ we have to choose $y(r)=r \times$ const. as expected. By fixing the scale for $r$ we can put this constant equal to 1 and we obtain

$\rho(t)=-\frac{3 K_{1} q_{0}}{a^{4}}$.

Analogously, the requirement that $n=n(t)$ leads, due to the first equation in (43), to

$n_{0}(r)=r^{2} n_{00}$

and therefore, due to (81) to

$\alpha(r)=r \alpha_{0}$

with

$-4 \pi G n_{00} \alpha_{0}=K_{1}$,

where $n_{00}$ and $\alpha_{0}$ are arbitrary constants.

To get for the cosmological equations (79) and (80) exactly the form derived in [11] for the nonrelativistic darkon fluid model (NDFM) we define the function $g(a(t))$ by

$g(a) \equiv 4 \pi G K_{1} \frac{q_{0}}{a}$.
Then the first equation in (43) as well as Eqs. (79) and (80) become

$n(t)=\frac{n_{00}}{a^{3}}, \quad \dot{g}=\frac{K_{1}}{a^{2}} \quad$ and $\quad \ddot{a}=\frac{g(a)}{a^{2}}$.

The equations given in (88) are identical with Eqs. (120)(122) in [11]. For the energy density (83) we obtain

$\rho(t)=-\frac{3 g}{4 \pi G a^{3}}$.

As shown in [11] the second and third equation in (88) give rise to two conserved quantities $K_{2,3}$

$K_{2}=\dot{a} K_{1}-\frac{1}{2} g^{2}$

and

$K_{3}=\frac{g^{3}}{6}+K_{2} g+\frac{K_{1}^{2}}{a}$.

These conservation laws lead for the choice $K_{2,3}>0$ (and consequently $K_{1}>0$ ) to a transition from an early decelerating phase of the Universe to a late accelerating phase with a transition redshift [11]

$1+z_{t}=\frac{K_{3}}{K_{1}^{2}}$.

What have we achieved?

We have shown that at sub-Hubble scales our general relativistic model agrees with our nonrelativistic model with the latter showing the observed transition from a decelerating to an accelerating phase of the Universe. But the observed transition redshift lies somewhere between $\frac{1}{2}$ and 1 (cp. [32]) which, at least for the $\Lambda \mathrm{CDM}$ model, corresponds to a luminosity distance of the order of the Hubble radius (cp. Fig. A 2.3 in [33]). So we are not yet able to prove this transition for our relativistic model. But what remains is an interesting result on the behavior of the spatial curvatures at sub-Hubble scales. To get this result we insert first of all our last results in to (70), respectively (71) and obtain

$b(t, r)=r^{2} K(t)$ with $K(t)=-\left(2 \ddot{a} a+a^{2}\right)$,

respectively,

$\dot{K}=-\frac{2 K_{1}}{a^{3}}$.

So the metric function $B(t, r)$ (69) becomes

$B^{2}(t, r)=\frac{a^{2}(t)}{1-r^{2} K(t)}$.

Next we show that $K(t)$ may be identified with the effective spatial curvature $K_{\text {eff }}$ introduced in (57).

First of all we insert the energy density $\rho$ from (89) into the third equation of (88) and obtain the standard cosmological 
RE equation with vanishing pressure

$\frac{3 \ddot{a}}{a}=-4 \pi G \rho$.

Eliminating $\ddot{a}$ in (93) by means of (96) we obtain the fundamental Friedmann equation [34],

$\dot{a}^{2}+K(t)=\frac{8 \pi G}{3} \rho a^{2}$,

but with a time-dependent spatial curvature $K(t)$, which agrees with the generalized Friedmann equation (72) if we identify at sub-Hubble scales

$K(t)=K_{\text {eff }}$.

For the sake of completeness we remark that the energy conservation equation (45) now takes the form

$\dot{\rho}+3 \frac{\dot{a}}{a} \rho-\frac{3}{8 \pi G a^{2}} \dot{K}=0$,

which is in agreement with its general form given in (61) (note that the shear $\sigma$ vanishes in the leading order).

Finally we may express $K(t)$ in terms of the function $g(a)$ which has been defined in (87) and can be determined by the solution of the cubic equation (91)

$K(a)=-\left(2 \frac{g(a}{a}+\frac{1}{K_{1}^{2}}\left(K-2+\frac{1}{2} g(a)^{2}\right)^{2}\right)$.

Let us summarize. At least the observed present-day accelerated expansion of the Universe is determined in our general relativistic model by a negative energy density [see Eq. (96)] or, equivalently, by a time-dependent spatial curvature [see Eq. (97)]. But the behavior of the spatial curvature at larger redshifts deserves for further studies.

Note that a time dependence of the spatial curvature with a possible sign change during evolution is already known for the Stephani solution of the EFEs [35-37].

\section{Non-comoving coordinates and modeling of halos}

In this section we consider the darkon fluid moving in the radial direction relative to the cosmic rest system (CRS). The metric in the CRS is assumed to be given by Schwarzschildlike coordinates. We will:

- derive the darkon fluid EOMs and the Einstein field equations by choosing the energy frame (vanishing heat flux) for the CRS (see [38]),

- look for weak-field solutions, which turn out to be equal to the nonrelativistic stationary solutions derived in [11] modeling halos.

\subsection{Cosmic rest system (CRS)}

Schwarzschild-like coordinates are defined by the spherically symmetric metric

$\mathrm{d} s^{2}=-\mathrm{e}^{2 \phi(t, r)} \mathrm{d} t^{2}+\mathrm{e}^{2 \lambda(t, r)} \mathrm{d} r^{2}+r^{2} \mathrm{~d} \Omega^{2}$.

This metric is assumed to be valid in the CRS defined by a time-like unit vector $n^{v}$ and a space-like unit vector $s^{v}$

$n^{\nu} \equiv\left(\mathrm{e}^{-\phi}, 0\right), \quad s^{\nu} \equiv\left(0, \mathrm{e}^{-\phi}\right)$

such that the CRS becomes the energy frame (vanishing heat flux) i.e. the EMT (39) takes in the CRS frame the form

$T^{\mu \nu}=\rho^{\star}(t, r) n^{\mu} n^{\nu}+p_{r}^{\star}(t, r) s^{\mu} s^{\nu}$,

where $\rho^{\star}$ and $p_{r}^{\star}$ are the energy density and the radial pressure in the CRS. Note that (103) contains no transversal pressure $p_{t}$ as (39) is free of it (radial movement does not change $p_{t}$ ).

The darkon fluid is assumed to move with velocity $v$ in the radial direction relative to the $\mathrm{CRS}$. Then the four-velocity $u^{\mu}$ and the vector $\theta^{\mu}$ are given by

$u^{\mu}=\gamma\left(n^{\mu}+v s^{\mu}\right), \quad \theta^{\mu}=\tilde{\theta} \gamma\left(v n^{\mu}+s^{\mu}\right)$,

where $\gamma(v) \equiv\left(1-v^{2}\right)^{-\frac{1}{2}}$.

Comparing (39) with (103) and using (104) we obtain

$\rho^{\star}=\frac{\rho}{1+v^{2}} \quad$ and $\quad p_{r}^{\star}=-\rho^{\star} v^{2}$

where $v(t, r)$ is determined by the requirement of the vanishing heat flux in the CRS

$\rho^{\star} v-n \tilde{\theta}=0$

\subsection{Einstein's field equations}

With the metric (101) and the EMT (103) we get for the Einstein field equations (see [39] and the literature cited therein)

$\kappa \rho^{\star}=\frac{1}{r^{2}}\left(r\left(1-\mathrm{e}^{-2 \lambda}\right)\right)^{\prime}$,

$\kappa p_{r}^{\star}=\frac{1}{r^{2}}\left(-1+\mathrm{e}^{-2 \lambda}\left(1+2 r \phi^{\prime}\right)\right)$,

$0=\phi^{\prime \prime}+\phi^{\prime 2}-\phi^{\prime} \lambda^{\prime}+\frac{\phi^{\prime}-\lambda^{\prime}}{r}$,

in which we had already used (110), and

$0=\dot{\lambda}$.

Using (110) in (107) we get immediately

$\dot{\rho}^{\star}=0$. 


\subsection{Darkon fluid EOMs}

From the darkon fluid EOMs (33-35) and (110) we get by using the metric (101) and Eq. (104) for $u^{\mu}$, respectively, $\theta^{\mu}$ :

- the continuity equation [first equation in (33)] becomes

$$
0=\mathrm{e}^{-\phi} \dot{n}+\mathrm{e}^{-\lambda} \frac{\left(r^{2} n v\right)^{\prime}}{r^{2}},
$$

- the Euler equation [second equation in (33)] becomes (cp. [39], Eq. (17))

$$
0=\mathrm{e}^{-\phi} \dot{v} \gamma^{2}+\mathrm{e}^{-\lambda}\left(v v^{\prime} \gamma^{2}+\phi^{\prime}\right)
$$

and

- by using $q^{\mu}=q \gamma\left(v n^{\mu}+s^{\mu}\right)$ we obtain from (34)

$$
\mathrm{e}^{-\phi} \gamma \dot{q}+\gamma \mathrm{e}^{-\lambda}\left(v q^{\prime}-q v^{\prime}\right)=\tilde{\theta}
$$

where, due to (35), $\tilde{\theta}$ obeys the EOM,

$$
\mathrm{e}^{\lambda}(\tilde{\theta} \gamma)+\left(\tilde{\theta} \gamma v \mathrm{e}^{\phi}\right)^{\prime}=0 .
$$

Finally we may express $\rho^{\star}$, defined by (40) and (105), in terms of the metric and the darkon fluid fields and we get

$\rho^{\star}=\frac{\gamma}{r^{2}} \mathrm{e}^{-\lambda}\left(r^{2} q n\right)^{\prime}$.

Sometimes it is useful to use instead of the darkon fluid EOMs the EOMs for $\rho^{\star}$ and $p_{r}^{\star}$, which follow from the covariant conservation of the EMT,

$\nabla_{\mu} T^{\mu v}=0$.

We recall that (117) can be derived either from the Bianchi identities for the Riemann tensor or directly from the darkon fluid EOMs. From the time-like part of (117) we reproduce (111) whereas the space-like part leads to the generalized Tolman-Oppenheimer-Volkoff (TOV) equation (see [40]), which in our case takes the form

$$
\left(\rho^{\star}+p_{r}^{\star}\right) \phi^{\prime}+2 \frac{p_{r}^{\star}}{r}+\left(p_{r}^{\star}\right)^{\prime}=0 .
$$

\section{Elimination of $\tilde{\theta}$, a conservation law}

By inserting $\tilde{\theta}$ from (106) into:

- Equation (115) and using (111), (113), and (118) we observe that (115) is identically satisfied.

- Equation (114) and using (116) for $\rho^{\star}$ we obtain

$$
\mathrm{e}^{-\phi} \dot{q}=\mathrm{e}^{-\lambda} \frac{q}{n r^{2}}\left(v n r^{2}\right)^{\prime}
$$

Combining (112) with (119) leads to the conservation law

$(n q)^{\cdot}=0$.

\subsection{Some exact relations}

Here we derive some exact expressions which follow from the coupled system of Einstein's field equations and the darkon fluid EOMs.

By using (110), (111), and (120) we conclude from (116) that

$\dot{v}=0$

and therefore the second equation in (105) leads to

$\dot{p}_{r}^{\star}=0$,

which, when used in the second Einstein equation (108) gives

$\dot{\phi}^{\prime}=0$.

Equation (123) can easily be integrated to give

$\phi(t, r)=\phi_{0}(r)+\phi_{1}(t)$,

where $\phi_{0}$ and $\phi_{1}$ are arbitrary functions of $r$ and $t$, respectively. Finally, by using (121) and (124), the Euler equation (113) can be integrated to give

$\phi_{0}(r)=\frac{1}{2} \log \left(1-v^{2}(r)\right)$.

6.5 Weak field limit for the Tolman-Oppenheimer-Volkoff (TOV) equation

By a weak-field limit we understand a space-time described by a small perturbation of the Minkowski metric at subHubble scales (cp. [41]).

To be specific we follow the procedure of Green and Wald [30] and put $(\epsilon \ll 1)$

$\phi=O(\epsilon), \quad \lambda=O(\epsilon), \quad v=O\left(\epsilon^{\frac{1}{2}}\right), \quad \partial_{r}=O\left(\epsilon^{-1}\right)$,

$\partial_{t}=O\left(\epsilon^{-\frac{1}{2}}\right)$.

Then we obtain in leading order:

- From (118)

$$
\rho \phi_{0}^{\prime}-2 \frac{\rho v^{2}}{r}-\left(\rho v^{2}\right)^{\prime}=0 .
$$

- From the second and third Einstein equation, (108) and (109),

$$
\lambda(r)=r \phi_{0}^{\prime}(r),
$$


which, when combined with the first Einstein equation (107), leads to the Poisson equation,

$$
4 \pi G \rho=\frac{1}{r^{2}}\left(r^{2} \phi_{0}^{\prime}\right)^{\prime} .
$$

- From (125)

$$
\phi_{0}=-\frac{1}{2} v^{2} .
$$

If we now insert (129) and (130) into (127) we obtain

$$
3\left(r^{2} \phi_{0}^{\prime}\right)^{\prime} \phi_{0}^{\prime}+2 \phi_{0}\left(r^{2} \phi_{0}^{\prime}\right)^{\prime}=0 \text {. }
$$

Multiplying (131) by $\left(-2 \phi_{0}\right)^{\frac{1}{2}}$ (integrating factor) we obtain

$$
\left(\left(-2 \phi_{0}\right)^{\frac{3}{2}}\left(r^{2} \phi_{0}^{\prime}\right)\right)^{\prime}=0,
$$

which, after integration, leads to the following nonlinear ordinary differential equation for the gravitational potential $(\beta=$ const.):

$$
\left(r^{2} \phi_{0}^{\prime}\right)^{\prime}=\frac{\beta}{2}\left(-2 \phi_{0}\right)^{-\frac{3}{2}}
$$

which was derived in [11] as the stationary solution of the spherically symmetric, nonrelativistic darkon fluid equations.

\subsection{Modeling halos}

In [11] we used the numerical solutions of (133) to determine the circular motion of a star in the potential $\phi_{0}$ given by the formula (see [42])

$\frac{\hat{v}^{2}(r)}{r}=\phi_{0}^{\prime}(r)$,

where $\hat{v}$ is the rotational velocity of the star. Thus, if all stars of a galaxy are in circular motion the graph of $\hat{v}$ gives the galactic rotation curve. We recall that the results reported in [11] are in qualitative agreement with observational data.

\section{Final remarks}

In this paper we have generalized our nonrelativistic darkon fluid model (NDFM), introduced in [8], and extended and reviewed in [11], to the framework of general relativity. Our relativistic model contains, as is the case for the NDFM, no free parameters in its Lagrangian. This feature distinguishes our model, to the best of our knowledge, from all other models for dark energy, respectively, dark matter. The relativistic model reproduces, at sub-Hubble scales, the cosmological equations derived from the NDFM (Sect. 5) and in the weakfield limit the nonlinear differential equation satisfied by the gravitational potential for stationary solutions of the NDFM (Sect. 6). We recall that the NDFM predicts qualitatively correct values of the late time cosmic acceleration as well as the flat behavior of galactic rotation curves $[8,11]$. Note that the derivation of already known results from approximate solutions of the relativistic model has led to new insights, respectively, physical interpretations: our nonrelativistic cosmological equations are different from the FLRW model. The cosmic acceleration is not attributed to a negative pressure (e.g. a positive cosmological constant) but it is due to a dynamically determined negative energy density. This property turns out to be equivalent to a time-dependent spatial curvature. In this regard, our relativistic model is very different from the model of dipolar dark matter and dark energy advocated by Blanchet and Tiec $[43,44]$. These authors consider in [43] a relativistic action which, to some extent, is equivalent to ours, but it differs mainly by the addition of an ad hoc internal force depending on the polarization field. This phenomenological internal force mimics a cosmological constant. Thus, their background model is the $\Lambda \mathrm{CDM}$ model, which is completely different from our model.

Finally we note that the comparison of our nonrelativistic model with the $\Lambda$ CDM model with $H(z)$ data (see Fig. 1. in [11]) suggests that it will be possible to discriminate between these two models only at larger redshifts.

We have managed to derive the nonrelativistic gravitational potential produced by a galactic halo in our model from a weak-field limit of the generalized TOV equation. But in contrast to the original application of TOV (hydrostatic equilibrium within a star) we have derived and applied the generalized TOV to a non-equilibrium situation given by non-comoving coordinates.

The main aim of the present paper was to present a general relativistic version of the NDFM and to look at its approximations which reproduce either the cosmological or the stationary solutions of the NDFM. This we have achieved but we are aware of the fact that further work on the consequences of the relativistic model is called for.

Open Access This article is distributed under the terms of the Creative Commons Attribution License which permits any use, distribution, and reproduction in any medium, provided the original author(s) and the source are credited.

Funded by $\mathrm{SCOAP}^{3}$ / License Version CC BY 4.0.

\section{References}

1. R. Jimenez (2013). arXiv:1307.2452. (to be publ. in: Cent. Eur. J. Phys.)

2. A. Joyce et al. (2014). arXiv:1407.0059 [astro-ph.CO]

3. M. Roos, J. Mod. Phys. 3, 1152 (2012). arXiv:1208.3662 [astroph.CO] 
4. J. Martin, Comptes Rendus-Phys. 13, 566 (2012). arXiv: 1205.3365 [gr-qc]

5. K. Garrett, G. Duda, Adv. Astron. Art. ID 968283 (2011)

6. D. Weinberg et al. (2013). arXiv:1306.0913 [astro-ph.CO]

7. P. Kroupa, PASA 29, 395 (2012). arXiv:1204.2546 [astro-ph.CO]

8. P.C. Stichel, W.J. Zakrzewski, Phys. Rev. D 80, 083513 (2009)

9. P.C. Stichel, W.J. Zakrzewski, Eur. Phys. J. C 70, 713 (2010)

10. P.C. Stichel, W.J. Zakrzewski, Int. J. Geom. Methods Mod. Phys. 9, 1261014 (2012)

11. P.C. Stichel, W.J. Zakrzewski, Entropy 15, 559 (2013)

12. M. Mathisson, Acta Phys. Pol. VI, 356 (1937)

13. J. Gleyzes et al. (2014). arXiv:1408.1952 [astro-ph.CO]

14. J. Weyssenhoff, A. Raabe, Acta Phys. Pol. IX, 7 (1947)

15. Th Dumitrescu, N. Seiberg, JHEP 1107, 095 (2011)

16. J. Frenkel, Zeits. f. Phys. 37, 243 (1926)

17. L. Costa, J. Natário (2014). arXiv:1410.6443 [gr-qc]

18. F. Hehl, Rep. Math. Phys. 9, 55 (1976)

19. F. Belinfante, Physica VII, 449 (1940)

20. N. Straumann, General Relativity, 2nd edn. (Springer, New York, 2013)

21. J. Ehlers, Gen. Relativ. Gravit. 25, 1225 (1993)

22. R. Maartens (1996). arXiv:astro-ph/9609119

23. S. Saha et al. (2014). arXiv: 1404.1220 [gr-qc]

24. C. Kolassis et al., Class. Quantum. Gravity 5, 1329 (1988)

25. S. Capozziello et al. arXiv: 1407.7293 [gr-qc]

26. S. Thirukkanesh et al., J. Math. Phys. 53, 032506 (2012)
27. C. Tsagas et al., Phys. Rep. 465, 61-147 (2008)

28. G. Ellis et al., Relativistic Cosmology (Cambridge University Press, Cambridge, 2013)

29. O. Bergmann, Phys. Lett. A 82, 383 (1981)

30. S. Green, R. Wald, Phys. Rev. 85, 063512 (2012)

31. P. Ivanov, Phys. Lett. B 680, 212 (2009)

32. O. Farooq, B. Ratra (2013). arXiv:1301.5243 [astro-ph.CO]

33. A. Liddle, An Introduction to Modern Cosmology (Wiley, New York, 2003)

34. St. Weinberg, Cosmology (University Press, Oxford, 2008)

35. H. Stephani, Commun. Math. Phys. 4, 137 (1967)

36. A. Krasinski, Gen. Relativ. Gravit. 15, 673 (1983)

37. A. Krasinski, Inhomogenous Cosmological Models (University Press, Cambridge, 1997)

38. G. Ballesteros et al., JCAP 1205, 038 (2012). arXiv:1112.4837

39. L. Herrera et al., Phys. Rev. 69, 084026 (2004)

40. R. Bowers et al., Astrophys. J. 188, 657 (1974)

41. T. Clifton et al., Phys. Rev. D 87, 063517 (2013). arXiv: 1210.0730 [gr-qc]

42. J. Binney, S. Tremaine, Galactic Dynamics (Princeton University Press, Princeton, 2008)

43. L. Blanchet, A. Tiec, Phys. Rev. D 80, 023524 (2009)

44. L. Blanchet et al. (2013). arXiv:1312.6991 [astro-ph.CO] 